University of Texas at El Paso

ScholarWorks@UTEP

$12-2016$

\title{
Optimal Group Decision Making Criterion and How It Can Help to Decrease Poverty, Inequality, and Discrimination
}

Vladik Kreinovich

The University of Texas at El Paso, vladik@utep.edu

Thongchai Dumrongpokaphan

Chiang Mai University, tcd43@hotmail.com

Follow this and additional works at: https://scholarworks.utep.edu/cs_techrep

Part of the Computer Sciences Commons

Comments:

Technical Report: UTEP-CS-16-104

\section{Recommended Citation}

Kreinovich, Vladik and Dumrongpokaphan, Thongchai, "Optimal Group Decision Making Criterion and How It Can Help to Decrease Poverty, Inequality, and Discrimination" (2016). Departmental Technical Reports (CS). 1075.

https://scholarworks.utep.edu/cs_techrep/1075

This Article is brought to you for free and open access by the Computer Science at ScholarWorks@UTEP. It has been accepted for inclusion in Departmental Technical Reports (CS) by an authorized administrator of ScholarWorks@UTEP.For more information, please contact Iweber@utep.edu. 


\title{
Optimal Group Decision Making Criterion and How It Can Help to Decrease Poverty, Inequality, and Discrimination
}

Vladik Kreinovich and Thongchai Dumrongpokaphoan

\begin{abstract}
Traditional approach to group decision making in economics is to maximize the GDP, i.e., the overall gain. The hope behind this approach is that the increased wealth will trickle down to everyone. Sometimes, this happens, but often, in spite of an increase in overall GDP, inequality remains: some people remain poor, some groups continue to face economic discrimination, etc. This shows that maximizing the overall gain is probably not always the best criterion in group decision making. In this chapter, we find a group decision making criterion which is optimal (in some reasonable sense), and we show that using this optimal criterion can indeed help to decrease poverty, inequality, and discrimination.
\end{abstract}

\section{Traditional Group Decision Making in Economics and Its Limitations: Formulation of the Problem}

Traditional approach to group decision making in economics. Traditional approach to group decision making in economics is to maximize the Gross Domestic Product (GDP), i.e., to maximize the overall gain $\sum_{i=1}^{n} g_{i}$, where $g_{i}$ is the gain of the $i$-th person.

The hope behind this approach. The hope behind the traditional approach is that the rising tide will lift all the boats, that the increased overall wealth will trickle down to everyone.

Vladik Kreinovich

Department of Computer Science, University of Texas at El Paso, 500 W. University

El Paso, Texas 79968, USA, e-mail: vladik@utep.edu

Thongchai Dumrongpokaphoan

Faculty of Mathematics, Faculty of Science, Chiang Mai University, Thailand

e-mail: tcd43@hotmail.com 
The reality does not not always follow this optimistic vision. Sometimes, the overall increase in wealth indeed increases everyone's income. However, often, in spite of an increase in overall GDP, inequality remains:

- some people remain poor,

- some groups continue to face economic discrimination, etc.

What can we conclude from this fact? The fact that maximizing the overall gain does not always lead to good consequences for everyone indicates that maximizing GDP is probably not always the best criterion in group decision making.

What we do in this paper. To improve the situation, let us try to find a better criterion for group decision making.

To come up with such a better criterion, we formulate the problem of selecting a criterion for group decision making as an optimization problem. We then solve this optimization problem, and we show that the resulting optimal group decision making criterion can indeed help in decreasing poverty, inequality, and discrimination.

\section{Individual decision Making: A Brief Reminder}

Need to consider individual decision making. In other to properly describe group decision making, it is necessary to first describe individual decision making.

Main assumptions behind the traditional decision theory. In this section, we will briefly describe the traditional decision theory; see, e.g., $[1,6,9]$. The main assumptions behind the traditional decision theory are as follows.

The first assumption is that for every two alternatives $a$ and $b$ :

- a person either prefers $a$ (we will denote it by $a \succ b$ ),

- or prefers $b(b \succ a)$,

- or for this person, alternatives $a$ and $b$ are of equal value (we will denote it by $a \sim b$ ).

The second assumption is that the person is rational, in the sense that this person's preferences are consistent:

- if $a \succ b$ and $b \succ c$, then $a \succ c$;

- if $a \sim b$ and $b \sim c$, then $a \sim c$;

- if $a \succ b$ and $b \sim c$, then $a \succ c$; and

- if $a \sim b$ and $b \succ c$, then $a \succ c$.

The main concept of the traditional decision theory: the notion of utility. Under the above assumptions, we can use the following idea to numerically describe the value of each alternative to a person. We select two alternatives:

- we select a very bad alternative $a_{0}$, which is much worse than anything that will be really proposed, and 
- we select a very good alternative $a_{1}$, which is much better than anything that will be really proposed.

Then, for every real number $p$ from the interval $[0,1]$, we can form a lottery $L(p)$ in which we get $a_{1}$ with probability $p$ and $a_{0}$ with the remaining probability $1-p$. The larger $p$, the better the lottery: if $p<p^{\prime}$, then $L(p) \prec L\left(p^{\prime}\right)$.

For each alternative $a$, and for every $p$, we either have $a \prec L(p)$ or $a \succ L(p)$ or $a \sim L(p)$. If $a \prec L(p)$ and $p<p^{\prime}$, then we will still have $a \prec L\left(p^{\prime}\right)$. Similarly, if we have $a \succ L(p)$ and $p>p^{\prime}$, then we will have $a \succ L\left(p^{\prime}\right)$. Thus, we have:

$$
\inf \{p: a \prec L(p)\}=\sup \{p: L(p) \prec a\} .
$$

This common probability value is called the utility of the alternative $a$. It is usually denoted by $u(a)$.

For the utility, for every $\varepsilon>0$, we have

$$
L(u(a)-\varepsilon) \prec a \prec L(u(a)+\varepsilon) .
$$

In this sense, $a$ is "almost" equivalent to to the lottery $L(u(a))$; we will denote this equivalence by $a \equiv L(u(a))$.

Utility is defined modulo a linear transformation. The numerical value of the utility depends on our selection of the alternatives $a_{0}$ and $a_{1}$. If we select a different pair $a_{0}^{\prime}$ and $a_{1}^{\prime}$, then we, in general, will get different numerical values of the corresponding utility $u^{\prime}(a)$. What is the relation between the new utility value $u^{\prime}(a)$ and the original utility value $u(a)$ ?

To answer this question, let us first consider the case when $a_{0} \prec a_{0}^{\prime} \prec a_{1}^{\prime} \prec a_{1}$. In this case, each of the new alternatives $a_{0}^{\prime}$ and $a_{1}^{\prime}$ is equivalent to an appropriate lottery $L(p): a_{0}^{\prime} \equiv L\left(u\left(a_{0}^{\prime}\right)\right)$ and $a_{1}^{\prime} \equiv L\left(u\left(a_{1}^{\prime}\right)\right)$ for some values $u\left(a_{0}^{\prime}\right)$ and $u\left(a_{0}^{\prime}\right)$.

By definition of the $\left(a_{0}^{\prime}, a_{1}^{\prime}\right)$ obased utility $u^{\prime}(a)$, the alternative $a$ is equivalent to the lottery $L^{\prime}\left(u^{\prime}(a)\right)$ in which we get $a_{1}^{\prime}$ with probability $u^{\prime}(a)$ and $a_{0}^{\prime}$ with the remaining probability $1-u^{\prime}(a)$.

If we replace $a_{1}^{\prime}$ with the equivalent lottery $L\left(a_{1}^{\prime}\right)$ and $a_{0}^{\prime}$ with the equivalent lottery $L\left(a_{0}^{\prime}\right)$, then we conclude that the original alternative $a$ is equivalent to the following 2-stage lottery:

- first, we select either 1 (with probability $u^{\prime}(a)$ ) or 0 (with probability $1-u^{\prime}(a)$ );

- then, depending on the value $i$ that we selected in the first stage, we select either $a_{1}$ (with probability $u\left(a_{1}^{\prime}\right)$ ) or $a_{0}$ (with probability $u\left(a_{0}^{\prime}\right)$ ).

As a result of this 2-stage lottery, we get either $a_{1}$ or $a_{0}$. By using the formula for the complete probability, we can find the probability $u(a)$ for selecting $a_{1}$ :

$$
u(a)=u^{\prime}(a) \cdot u\left(a_{1}^{\prime}\right)+\left(1-u^{\prime}(a)\right) \cdot u\left(a_{0}^{\prime}\right) .
$$

Thus, the original alternative $a$ is equivalent to the lottery in which we select $a_{1}$ with this probability $u(a)$ and $a_{0}$ with the remaining probability $1-u(a)$. So, $a \equiv L(u(a))$.

The above formula shows that the utilities $u(a)$ and $u^{\prime}(a)$ are connected by a linear dependence. This was when we have $a_{0} \prec a_{0}^{\prime} \prec a_{1}^{\prime} \prec a_{1}$. 
In the general case, when we want to find a relation between utilities $u(a)$ and $u^{\prime}(a)$ corresponding to different pairs $\left(a_{0}, a_{1}\right)$ and $\left(a_{0}^{\prime}, a_{1}^{\prime}\right)$, the way to find this connection is by considering a third pair $\left(a_{0}^{\prime \prime}, a_{1}^{\prime \prime}\right)$ for which $a_{0}^{\prime \prime} \prec a_{0}, a_{0}^{\prime \prime} \prec a_{0}^{\prime}, a_{1} \prec a_{1}^{\prime \prime}$, and $a_{1}^{\prime} \prec a_{1}^{\prime \prime}$. In this case, the above argument shows that:

- $u^{\prime \prime}(a)$ is a linear function of $u^{\prime}(a)$, and

- $u^{\prime \prime}(a)$ is a linear function of $u(a)$ - thus, $u(a)$ is a linear function of $u^{\prime \prime}(a)$.

Substituting the linear expression of $u^{\prime \prime}(a)$ in terms of $u^{\prime}(a)$ into the linear dependence of $u(a)$ on $u^{\prime \prime}(a)$ and using the fact that a composition of two linear functions is linear, we conclude that $u^{\prime}(a)$ is indeed a linear function of $u(a)$;

$$
u^{\prime}(a)=k \cdot u(a)+\ell
$$

for some $k>0$ and $\ell$.

Now, we have all the preliminaries needed to start discussing group decision making.

\section{Group Decision Making: Analysis of the Problem}

What is a group decision making criterion? To compare several alternatives, we need to know how valuable is each alternative to each person from the group.

The value of each alternative $a$ to each person $i$ can be described by the $i$-th utility $u_{i}(a)$ of this alternative. Thus, to describe the value of the alternative $a$ to the group, we can use the tuple $u(a)=\left(u_{1}(a), \ldots, u_{n}(a)\right)$ consisting of all these utility values.

As we have mentioned earlier, for each person $i$, his or her utility values are defined modulo arbitrary linear transformation: $u_{i}(a) \rightarrow k_{i} \cdot u_{i}(a)+\ell_{i}$. We are considering situations in which we start with a certain status quo $s_{0}$. It is therefore reasonable to re-scale utilities in such a way that the status-quo value of utility corresponds to 0 . In other words, instead of the original utility values, we consider the utility gains $u_{i}(a)-u_{i}\left(s_{0}\right)$.

In this re-scaled case, for each alternative, we have $u_{i}(a)>0$ : if $u_{i}(a)<0$, i.e., if the $i$-th person loses with the adoption of the alternative $a$, this person would then never agree to this alternative. Similarly, if $u_{i}(a)=0$, i.e., if the person does not gain anything in the alternative $a$, why would this person participate in making this decision?

Thus, the only tuples that we have to consider are tuples $u(a)$ in which all the components $u_{i}(a)$ are positive.

For every two alternatives $a$ and $b$, based on the corresponding tuples, we need to decide:

- whether $a$ is better than $b$,

- or $b$ is better than $a$,

- or that for the group, the alternatives $a$ and $b$ are equivalent. 
Such a decision is what we call a group decision making criterion.

Of course, if everyone benefits, i.e., if $u_{i}<u_{i}^{\prime}$ for all $i$, then clearly, the tuple $u^{\prime}$ is better than the tuple $u$. Similarly, if no one loses, i.e., if $u_{i} \leq u_{i}^{\prime}$ for all $i$, then the tuple $u^{\prime}$ should be either better or of the same quality as $u$-but not worse.

Finally, a group decision making criterion should be continuous in the following sense: if we have $a^{(k)} \rightarrow a$ for some sequence $a^{(k)}$, and if for every $k$, we have $a^{(k)} \succ b$ or $a^{(k)} \sim b$, then for the limit sequence $a$, we should also have $a \prec b$ or $a \sim b$.

In other words, if a tuple $a$ is worse than a tuple $b$, then all the tuples in some neighborhood of $a$ should also be worse than $b$.

Let us describe all this in precise terms.

Definition 1. By a pre-ordering relation on a set $A$, we mean a pair of binary relations $\prec$ and $\sim$ for which $a \prec b$ for some $a$ and $b$ and for which the following properties hold for all $a, b$, and $c$ :

- $a \sim a$ and $a \nprec a$;

- if $a \prec b$ and $b \prec c$, then $a \prec c$;

- if $a \sim b$ then $b \sim a$;

- if $a \sim b$ and $b \sim c$, then $a \sim c$;

- if $a \prec b$ and $b \sim c$, then $a \prec c$;

- if $a \sim b$ and $b \prec c$, then $a \prec c$.

Definition 2. A pre-ordering is called linear (or total) if for every two elements a and $b$, we have either $a \prec b$, or $b \prec a$, or $a \sim b$.

Definition 3. By a group decision making criterion, we mean a linear (total) preordering $(\prec, \sim)$ on the set $\mathbb{R}_{+}^{n}$ of all possible $n$-tuples $u=\left(u_{1}, \ldots, u_{n}\right)$ of positive real numbers for which:

- if $u_{i}<u_{i}^{\prime}$ for all $i$, then $u \prec u^{\prime}$,

- if $u_{i} \leq u_{i}^{\prime}$ for all $i$, then either $u \prec u^{\prime}$ or $u \sim u^{\prime}$, and

- if $a \prec b$, then for all the tuples $a^{\prime}$ from some neighborhood of $a$, we should also have $a^{\prime} \prec b$.

\section{We Want to Find The Best Group Decision Making Criterion}

Discussion. To select the best group decision making criterion, we need to be able to compare different criteria: some criteria are better, some are worse, some are equivalent. In other words, we need to describe a linear pre-ordering $(<, \equiv)$ on the set of all possible criteria.

Then, we should select the best (optimal) criterion $A$, i.e., a criterion for which, for every other criterion $B$, we should have either $B<A$ or $B \equiv A$.

It is reasonable to require that there should be only one best criterion $A$. Indeed, if no criterion is the best, then this pre-ordering is of no use. On the other hand, if several criteria are the best, this means that our pre-ordering is not final: we can us this 
non-uniqueness to optimize something else. For example, if several different group decision making criteria are of equal value based on the economic consequences, it is reasonable to select the only which is the easiest to compute. This means that the original comparison $<$ (based only on economical consequences) is now replaced by a more complex comparison $a<^{\prime} b$ which holds if:

- either $b$ is better economically,

- or, economically, $a$ and $b$ are of the same quality, but $b$ is easier to compute.

Eventually, we will thus reach the final situation, in which for the resulting linear pre-ordering $(<, \equiv)$, exactly one group decision making criterion is the best.

Another reasonable requirement is that the comparison between different group decision making criteria should not change if we simply re-scale the individual utility values, i.e., replace the original utility values $u_{i}$ by re-scaled values $u_{i}^{\prime}=k_{i} \cdot u_{i}$.

Finally, it is reasonable to require that all participants are equal, in the sense that nothing should change if we simply swap two or more participants (and their utilities).

Thus, we arrive at the following definitions.

Definition 4. By a comparison between group decision making criteria, we mean a linear (total) pre-ordering $(<, \equiv)$ on the set of all possible group decision making criteria.

Definition 5. We say that a group decision making criterion A is optimal with respect to a given comparison $(<, \equiv)$ if for every other group decision making criterion $B$, we have either $B<A$, or $B \equiv A$.

Definition 6. We say that a comparison $(<, \equiv)$ is final if there exists exactly one group decision making criterion which is optimal with respect to this comparison.

Definition 7. Let $k=\left(k_{1}, \ldots, k_{n}\right)$ be a tuple of positive numbers $k_{i}>0$.

- For every tuple $u=\left(u_{1}, \ldots, u_{n}\right)$, by its $k$-rescaling, we mean a tuple

$$
S_{k}(u)=\left(k_{1} \cdot u_{1}, \ldots, k_{n} \cdot u_{n}\right) .
$$

- By a $k$-rescaling $S_{k}(A)=\left(\prec_{k}, \sim_{k}\right)$ of a group decision making criterion

$$
A=(\prec, \sim),
$$

we mean the following criterion:

$$
a \prec_{k} b \Leftrightarrow S_{k}(a) \prec S_{k}(b) \text {, and } a \sim_{k} b \Leftrightarrow S_{k}(a) \sim S_{k}(b) .
$$

Definition 8. We say that a comparison $(<, \equiv)$ is scale-invariant if for every tuple $k$ and for every two group decision making criteria $A$ and $B$, we have:

$$
\begin{gathered}
A<B \Leftrightarrow S_{k}(A)<S_{k}(B) ; \text { and } \\
A \equiv B \Leftrightarrow S_{k}(A) \equiv S_{k}(B) .
\end{gathered}
$$


Definition 9. Let $\pi:\{1, \ldots, n\} \rightarrow\{1, \ldots, n\}$ be a permutation.

- For every tuple $u=\left(u_{1}, \ldots, u_{n}\right)$, by its permutation, we mean a tuple

$$
\pi(u)=\left(u_{\pi(1)}, \ldots, u_{\pi(n)}\right) .
$$

- By a permutation $\pi(A)=\left(\prec_{\pi}, \sim_{\pi}\right)$ of a group decision making criterion

$$
A=(\prec, \sim),
$$

we mean the following criterion:

$$
a \prec_{\pi} b \Leftrightarrow \pi(a) \prec \pi(b), \text { and } a \sim_{\pi} b \Leftrightarrow \pi(a) \sim \pi(b) .
$$

Definition 10. We say that a comparison $(<, \equiv)$ is permutation-invariant iffor every permutation $\pi$ and for every two group decision making criteria $A$ and $B$, we have:

$$
\begin{gathered}
A<B \Leftrightarrow \pi(A)<\pi(B) ; \text { and } \\
A \equiv B \Leftrightarrow \pi(A) \equiv \pi(B) .
\end{gathered}
$$

Main Result. For every final scale-invariant permutation-invariant comparison

$$
(<, \equiv),
$$

the optimal group decision making $(\prec, \sim)$ has the following form:

$$
\begin{gathered}
a=\left(a_{1}, \ldots, a_{n}\right) \prec b=\left(b_{1}, \ldots, b_{n}\right) \Leftrightarrow \prod_{i=1}^{n} a_{i}<\prod_{i=1}^{n} b_{i} ; \text { and } \\
a=\left(a_{1}, \ldots, a_{n}\right) \sim b=\left(b_{1}, \ldots, b_{n}\right) \Leftrightarrow \prod_{i=1}^{n} a_{i}=\prod_{i=1}^{n} b_{i} .
\end{gathered}
$$

Comment. For reader's convenience, the proof of this result is given in a special Appendix.

Discussion. According to our result, according to the optimal group decision making criterion, we should select an alternative $a$ for which the product $\prod_{i=1}^{n} u_{i}(a)$ of all the utilities attains the largest possible value.

This group decision making criterion was first proposed by the Nobelist John Nash [5] (see also [4]) and is thus known as Nash's bargaining solution. Thus, what we prove is that Nash's bargaining solution is the optimal groul decision making criterion. 


\section{The Resulting Optimal Criterion for Group Decision Making Is In Good Accordance with Common Sense}

The objective of group decision making is to make sure that the first person is happy, and that the second person is happy, and that the their person is happy, etc. From this viewpoint, selecting the best alternative means selecting the alternative for which the degree to which first person is happy and the second person is happy, etc., is the largest possible.

In line with the usual fuzzy logic techniques (see, e.g., $[3,8,10])$, to describe the degree to which this objective is satisfied, we must:

- first, find the degrees to which each person is happy, and then,

- use an "and"-operation (t-norm) $f_{\&}(a, b)$ to combine these degrees.

For each alternative $a$, a reasonable measure of the degree $d_{i}(a)$ to which the $i$-th person is happy with this alternative is his/her utility $u_{i}(a)$. It is therefore reasonable to assume that the degree $d_{i}(a)$ can be determined if we know the utility $u_{i}(a)$, i.e., that $d_{i}(a)=f\left(u_{i}(a)\right)$ for an appropriate algorithm $f(x)$.

The simplest possible case is when we simply take $d_{i}(a)=u_{i}(a)$, with $f(x)=x$. Among the simplest - and most widely used - t-norms is the algebraic product $f_{\&}(a, b)=a \cdot b$. If we use this t-norm, then the degree to which all the members of the groups are happy with the given alternative $a$ is equal to the product

$$
\prod_{i=1}^{n} d_{i}(a)=\prod_{i=1}^{n} u_{i}(a) .
$$

In this case, selecting the best alternative means selecting the alternative $a$ for which this product attains its largest possible value.

This is exactly Nash's bargaining solution which, as we have shown, is the optimal group decision making criterion. Thus, Nash's bargaining solution is indeed in good accordance with common sense - namely, it is in good accordance with the simplest possible formalization of the above common sense criterion.

\section{The Resulting Optimal Criterion for Group Decision Making Can Help in Decreasing Poverty, Inequality, and Discrimination}

What happens now: it is possible that maximizing GDP retains poverty, inequality, and discrimnination. The problem with the current economic development - which is motivated by the need to maximize the GDP - is that while the GDP is increased and some people get richer, some other people remain at the same poverty level as before. 
In other words, while for some people, the gain $g_{i}$ is positive, for many others, the gain is practically zero: $g_{j} \approx 0$. Thus, the increase in GDP sometimes increases inequality as well - the rich get richer, but the poor remain equally poor.

Poverty is rarely distributed uniformly: usually, some population groups are poorer. When the income of such groups does not increase, this is an example of an economic discrimination.

How the use of the new criterion for group decision making can help prevent such situations. We would like to avoid the situations in which for the selected alternative $a$, at least one person $j$ does not get any increase in utility, i.e., $u_{j}(a) \approx 0$ for some $j$. Let us show that such alternatives are indeed avoided.

Indeed, if for some alternative $a$, we have $u_{j}(a)$ for some person $j$, then for this alternative $a$, the product of utilities $\prod_{i=1}^{n} u_{i}$ is practically equal to $0-$ i.e., to its smallest possible value. A simple redistribution in which the $j$-th person (and other people who initially got practically nothing) would get a little more will immediately increase the product.

So, when we select an alternative for which the product of utilities is the largest possible, we will never select an alternative in which one of the persons practically does not get any increase in utility.

In this sense, the new criterion helps decrease inequality. And if there is a whole group of people which is discriminated against - e.g., for which $u_{j} \approx 0$ for all persons $j$ from this group - then the corresponding alternative will never be selected if we use the new group decision making criterion.

\section{Acknowledgments}

This work was supported by Chiang Mai University, Thailand. This work was also supported in part by the National Science Foundation grants HRD-0734825 and HRD-1242122 (Cyber-ShARE Center of Excellence) and DUE-0926721, and by an award "UTEP and Prudential Actuarial Science Academy and Pipeline Initiative" from Prudential Foundation.

\section{References}

1. P. C. Fishburn, Utility Theory for Decision Making, John Wiley \& Sons Inc., New York, 1969.

2. A. Jaimes, C. Tweedy, T. Magoc, V. Kreinovich, and M. Ceberio, "Selecting the Best Location for a Meteorological Tower: A Case Study of Multi-Objective Constraint Optimization", Journal of Uncertain Systems, 2010, Vol. 4, No. 4, pp. 261-269.

3. G. Klir and B. Yuan, Fuzzy Sets and Fuzzy Logic, Prentice Hall, Upper Saddle River, New Jersey, 1995.

4. R. D. Luce and R. Raiffa, Games and Decisions: Introduction and Critical Survey, Dover, New York, 1989. 
5. J. Nash, "Two-Person Cooperative Games," Econometrica, 1953, Vol. 21, pp. 128-140.

6. H. T. Nguyen, O. Kosheleva, and V. Kreinovich, "Decision making beyond Arrow's 'impossibility theorem', with the analysis of effects of collusion and mutual attraction", International Journal of Intelligent Systems, 2009, Vol. 24, No. 1, pp. 27-47.

7. H. T. Nguyen and V. Kreinovich, Applications of Continuous Mathematics to Computer Science, Kluwer, Dordrecht, 1997.

8. H. T. Nguyen and E. A. Walker, A First Course in Fuzzy Logic, Chapman and Hall/CRC, Boca Raton, Florida, 2006.

9. H. Raiffa, Decision Analysis, Addison-Wesley, Reading, Massachusetts, 1970.

10. L. A. Zadeh, "Fuzzy sets", Information and Control, 1965, Vol. 8, pp. 338-353.

\section{Proof of the Main Result}

$1^{\circ}$. In this proof, we will use ideas first described in [2] and in [7].

$2^{\circ}$. Let us first prove that for every every final scale-invariant permutation-invariant comparison $(<, \equiv)$, the optimal group decision making $A=(\prec, \sim)$ it itself scaleand permutation-invariant, i.e., $A=S_{k}(A)$ for every tuple $k$ and $\pi(A)=A$ for every permutation $A$.

Indeed, let us prove that $A=S_{k}(A)$ for every tuple $k=\left(k_{1}, \ldots, k_{n}\right)$. Let us denote $k^{-1}=\left(k_{1}^{-1}, \ldots, k_{n}^{-1}\right)$; then, clearly, $S_{k}\left(S_{k^{-1}}(B)\right)=B$ for all $B$. Since the group decision making criterion $A$ is optimal, then for every group decision making $B$, we have $S_{k^{-1}}(B)<A$ or $S_{k^{-1}}(B) \equiv A$. Since the comparison $(<, \equiv)$ is scaleinvariant, $S_{k^{-1}}(B)<A$ implies that $S_{k}\left(S_{k^{-1}}(B)\right)<S_{k}(A)$, i.e., that $B<S_{k}(A)$. Similarly, $S_{k^{-1}}(B) \equiv A$ implies that $S_{k}\left(S_{k^{-1}}(B)\right) \equiv S_{k}(A)$, i.e., that $B \equiv S_{k}(A)$.

Thus, for every group decision making criterion $B$, we have either $B<S_{k}(A)$ or $B \equiv S_{k}(A)$. By definition, this means that the group decision criterion $S_{k}(A)$ is optimal. However, our comparison $(<, \equiv)$ is final. Thus, there should be only one optimal group decision making criterion. So, we conclude that $S_{k}(A)=A$.

Similarly, we can prove that $\pi(A)=A$ for any permutation $\pi$. Let us denote the inverse permutation by $\pi^{-1}$; then, clearly, $\pi\left(\pi^{-1}(B)\right)=B$ for all $B$. Since the group decision making criterion $A$ is optimal, then for every group decision making $B$, we have $\pi^{-1}(B)<A$ or $\pi^{-1}(B) \equiv A$. Since the comparison $(<, \equiv)$ is permutationinvariant, $\pi^{-1}(B)<A$ implies that $\pi\left(\pi^{-1}(B)\right)<\pi(A)$, i.e., that $B<\pi(A)$. Similarly, $\pi^{-1}(B) \equiv A$ implies that $\pi\left(\pi^{-1}(B)\right) \equiv \pi(A)$, i.e., that $B \equiv \pi(A)$.

Thus, for every group decision making criterion $B$, we have either $B<\pi(A)$ or $B \equiv \pi(A)$. By definition, this means that the group decision criterion $\pi(A)$ is optimal. However, our comparison $(<, \equiv)$ is final. Thus, there should be only one optimal group decision making criterion. So, we conclude that $\pi(A)=A$.

$3^{\circ}$. Let us now use the scale-invariance of the optimal group decision making criterion $(\prec, \sim)$. Due to this scale-invariance, for every $y_{1}, \ldots, y_{n}, y_{1}^{\prime}, \ldots, y_{n}^{\prime}$, we can take $\lambda_{i}=\frac{1}{y_{i}}$ and conclude that

$$
\left(y_{1}^{\prime}, \ldots, y_{n}^{\prime}\right) \sim\left(y_{1}, \ldots, y_{n}\right) \Leftrightarrow\left(\frac{y_{1}^{\prime}}{y_{1}}, \ldots, \frac{y_{n}^{\prime}}{y_{n}}\right) \sim(1, \ldots, 1) .
$$


Thus, to describe the equivalence relation $\sim$, it is sufficient to describe the set of all the vectors $z=\left(z_{1}, \ldots, z_{n}\right)$ for which $z \sim(1, \ldots, 1)$. Similarly,

$$
\left(y_{1}^{\prime}, \ldots, y_{n}^{\prime}\right) \succ\left(y_{1}, \ldots, y_{n}\right) \Leftrightarrow\left(\frac{y_{1}^{\prime}}{y_{1}}, \ldots, \frac{y_{n}^{\prime}}{y_{n}}\right) \succ(1, \ldots, 1) .
$$

Thus, to describe the ordering relation $\succ$, it is sufficient to describe the set of all the vectors $z=\left(z_{1}, \ldots, z_{n}\right)$ for which $z \succ(1, \ldots, 1)$.

Alternatively, we can take $\lambda_{i}=\frac{1}{y_{i}^{\prime}}$ and conclude that

$$
\left(y_{1}^{\prime}, \ldots, y_{n}^{\prime}\right) \succ\left(y_{1}, \ldots, y_{n}\right) \Leftrightarrow(1, \ldots, 1) \succ\left(\frac{y_{1}}{y_{1}^{\prime}}, \ldots, \frac{y_{n}}{y_{n}^{\prime}}\right) .
$$

Thus, it is also sufficient to describe the set of all the vectors $z=\left(z_{1}, \ldots, z_{n}\right)$ for which $(1, \ldots, 1) \succ z$.

$4^{\circ}$. The above equivalence involves division. To simplify the description, we can take into account that in the logarithmic space, division becomes a simple difference: $\ln \left(\frac{y_{i}^{\prime}}{y_{i}}\right)=\ln \left(y_{i}^{\prime}\right)-\ln \left(y_{i}\right)$. To use this simplification, let us consider the logarithms $Y_{i} \stackrel{\text { def }}{=} \ln \left(y_{i}\right)$ of different values. In terms of these logarithms, the original values can be reconstructed as $y_{i}=\exp \left(Y_{i}\right)$. In terms of these logarithms, we thus need to consider:

- the set $S_{\sim}$ of all the tuples $Z=\left(Z_{1}, \ldots, Z_{n}\right)$ for which

$$
z=\left(\exp \left(Z_{1}\right), \ldots, \exp \left(Z_{n}\right)\right) \sim(1, \ldots, 1),
$$

and

- the set $S_{\succ}$ of all the tuples $Z=\left(Z_{1}, \ldots, Z_{n}\right)$ for which

$$
z=\left(\exp \left(Z_{1}\right), \ldots, \exp \left(Z_{n}\right)\right) \succ(1, \ldots, 1) .
$$

We will also consider the set $S_{\prec}$ of all the tuples $Z=\left(Z_{1}, \ldots, Z_{n}\right)$ for which $(1, \ldots, 1) \succ z=\left(\exp \left(Z_{1}\right), \ldots, \exp \left(Z_{n}\right)\right)$. Since the pre-ordering relation $(\prec, \sim)$ is linear (total), for every tuple $z$ :

- either $z \sim(1, \ldots, 1)$,

- or $z \succ(1, \ldots, 1)$,

- or $(1, \ldots, 1) \succ z$.

In particular, this is true for $z=\left(\exp \left(Z_{1}\right), \ldots, \exp \left(Z_{n}\right)\right)$. Thus, for every tuple $Z$ :

- either $Z \in S_{\sim}$,

- or $Z \in S_{\succ}$,

- or $Z \in S_{\prec}$. 
$5^{\circ}$. Let us prove that the set $S_{\sim}$ is closed under addition, i.e., that if the tuples $Z=$ $\left(Z_{1}, \ldots, Z_{n}\right)$ and $Z^{\prime}=\left(Z_{1}^{\prime}, \ldots, Z_{n}^{\prime}\right)$ belong to the set $S_{\sim}$, then their component-wise sum

$$
Z+Z^{\prime}=\left(Z_{1}+Z_{1}^{\prime}, \ldots, Z_{n}+Z_{n}^{\prime}\right)
$$

also belongs to the set $S_{\sim}$.

Indeed, by definition of the set $S_{\sim}$, the condition $Z \in S_{\sim}$ means that

$$
\left(\exp \left(Z_{1}\right), \ldots, \exp \left(Z_{n}\right)\right) \sim(1, \ldots, 1) .
$$

Using scale-invariance with $\lambda_{i}=\exp \left(Z_{i}^{\prime}\right)$, we conclude that

$$
\left(\exp \left(Z_{1}\right) \cdot \exp \left(Z_{1}^{\prime}\right), \ldots, \exp \left(Z_{n}\right) \cdot \exp \left(Z_{n}^{\prime}\right)\right) \sim\left(\exp \left(Z_{1}^{\prime}\right), \ldots, \exp \left(Z_{n}^{\prime}\right)\right) .
$$

On the other hand, the condition $Z^{\prime} \in S_{\sim}$ means that

$$
\left(\exp \left(Z_{1}^{\prime}\right), \ldots, \exp \left(Z_{n}^{\prime}\right)\right) \sim(1, \ldots, 1) .
$$

Thus, due to transitivity of the equivalence relation $\sim$, we conclude that

$$
\left(\exp \left(Z_{1}\right) \cdot \exp \left(Z_{1}^{\prime}\right), \ldots, \exp \left(Z_{n}\right) \cdot \exp \left(Z_{n}^{\prime}\right)\right) \sim(1, \ldots, 1) .
$$

Since for every $i$, we have $\exp \left(Z_{i}\right) \cdot \exp \left(Z_{i}^{\prime}\right)=\exp \left(Z_{i}+Z_{i}^{\prime}\right)$, we thus conclude that

$$
\left(\exp \left(Z_{1}+Z_{1}^{\prime}\right), \ldots, \exp \left(Z_{n}+Z_{n}^{\prime}\right)\right) \sim(1, \ldots, 1) .
$$

By definition of the set $S_{\sim}$, this means that the tuple $Z+Z^{\prime}$ belongs to the set $S_{\sim}$.

$6^{\circ}$. Similarly, we can prove that the set $S_{\succ}$ is closed under addition, i.e., that if the tuples $Z=\left(Z_{1}, \ldots, Z_{n}\right)$ and $Z^{\prime}=\left(Z_{1}^{\prime}, \ldots, Z_{n}^{\prime}\right)$ belong to the set $S_{\succ}$, then their component-wise sum

$$
Z+Z^{\prime}=\left(Z_{1}+Z_{1}^{\prime}, \ldots, Z_{n}+Z_{n}^{\prime}\right)
$$

also belongs to the set $S_{\succ}$.

Indeed, by definition of the set $S_{\succ}$, the condition $Z \in S_{\succ}$ means that

$$
\left(\exp \left(Z_{1}\right), \ldots, \exp \left(Z_{n}\right)\right) \succ(1, \ldots, 1) .
$$

Using scale-invariance with $\lambda_{i}=\exp \left(Z_{i}^{\prime}\right)$, we conclude that

$$
\left(\exp \left(Z_{1}\right) \cdot \exp \left(Z_{1}^{\prime}\right), \ldots, \exp \left(Z_{n}\right) \cdot \exp \left(Z_{n}^{\prime}\right)\right) \succ\left(\exp \left(Z_{1}^{\prime}\right), \ldots, \exp \left(Z_{n}^{\prime}\right)\right) .
$$

On the other hand, the condition $Z^{\prime} \in S_{\succ}$ means that

$$
\left(\exp \left(Z_{1}^{\prime}\right), \ldots, \exp \left(Z_{n}^{\prime}\right)\right) \succ(1, \ldots, 1) .
$$

Thus, due to transitivity of the strict preference relation $\succ$, we conclude that 


$$
\left(\exp \left(Z_{1}\right) \cdot \exp \left(Z_{1}^{\prime}\right), \ldots, \exp \left(Z_{n}\right) \cdot \exp \left(Z_{n}^{\prime}\right)\right) \succ(1, \ldots, 1) .
$$

Since for every $i$, we have $\exp \left(Z_{i}\right) \cdot \exp \left(Z_{i}^{\prime}\right)=\exp \left(Z_{i}+Z_{i}^{\prime}\right)$, we thus conclude that

$$
\left(\exp \left(Z_{1}+Z_{1}^{\prime}\right), \ldots, \exp \left(Z_{n}+Z_{n}^{\prime}\right)\right) \succ(1, \ldots, 1) .
$$

By definition of the set $S_{\succ}$, this means that the tuple $Z+Z^{\prime}$ belongs to the set $S_{\succ}$.

$7^{\circ}$. A similar argument shows that the set $S_{\prec}$ is closed under addition, i.e., that if the tuples $Z=\left(Z_{1}, \ldots, Z_{n}\right)$ and $Z^{\prime}=\left(Z_{1}^{\prime}, \ldots, Z_{n}^{\prime}\right)$ belong to the set $S_{\prec}$, then their component-wise sum

$$
Z+Z^{\prime}=\left(Z_{1}+Z_{1}^{\prime}, \ldots, Z_{n}+Z_{n}^{\prime}\right)
$$

also belongs to the set $S_{\prec}$.

$8^{\circ}$. Let us now prove that the set $S_{\sim}$ is closed under the "unary minus" operation, i.e., that if $Z=\left(Z_{1}, \ldots, Z_{n}\right) \in S_{\sim}$, then $-Z \stackrel{\text { def }}{=}\left(-Z_{1}, \ldots,-Z_{n}\right)$ also belongs to $S_{\sim}$.

Indeed, $Z \in S_{\sim}$ means that

$$
\left(\exp \left(Z_{1}\right), \ldots, \exp \left(Z_{n}\right)\right) \sim(1, \ldots, 1)
$$

Using scale-invariance with $\lambda_{i}=\exp \left(-Z_{i}\right)=\frac{1}{\exp \left(Z_{i}\right)}$, we conclude that

$$
(1, \ldots, 1) \sim\left(\exp \left(-Z_{1}\right), \ldots, \exp \left(-Z_{n}\right)\right)
$$

i.e., that $-Z \in S_{\sim}$.

$9^{\circ}$. Let us prove that if $Z=\left(Z_{1}, \ldots, Z_{n}\right) \in S_{\succ}$, then $-Z \stackrel{\text { def }}{=}\left(-Z_{1}, \ldots,-Z_{n}\right)$ belongs to $S_{\prec}$.

Indeed, $Z \in S_{\succ}$ means that

$$
\left(\exp \left(Z_{1}\right), \ldots, \exp \left(Z_{n}\right)\right) \succ(1, \ldots, 1)
$$

Using scale-invariance with $\lambda_{i}=\exp \left(-Z_{i}\right)=\frac{1}{\exp \left(Z_{i}\right)}$, we conclude that

$$
(1, \ldots, 1) \succ\left(\exp \left(-Z_{1}\right), \ldots, \exp \left(-Z_{n}\right)\right)
$$

i.e., that $-Z \in S_{\prec}$.

Similarly, we can show that if $Z \in S_{\prec}$, then $-Z \in S_{\succ}$.

$10^{\circ}$. From Part 5 of this proof, it now follows that if $Z=\left(Z_{1}, \ldots, Z_{n}\right) \in S_{\sim}$, then $Z+Z \in S_{\sim}$, that $Z+(Z+Z) \in S_{\sim}$, etc., i.e., that for every positive integer $p$, the tuple

$$
p \cdot Z=\left(p \cdot Z_{1}, \ldots, p \cdot Z_{n}\right)
$$

also belongs to the set $S_{\sim}$. 
By using Part 8, we can also conclude that this is true for negative integers $p$ as well. Finally, by taking into account that the zero tuple $0 \stackrel{\text { def }}{=}(0, \ldots, 0)$ can be represented as $Z+(-Z)$, we conclude that $0 \cdot Z=0$ also belongs to the set $S_{\sim}$.

Thus, if a tuple $Z$ belongs to the set $S_{\sim}$, then for every integer $p$, the tuple $p \cdot Z$ also belongs to the set $S_{\sim}$.

$11^{\circ}$. Similarly, from Parts 6 and 7 of this proof, it follows that

- if $Z=\left(Z_{1}, \ldots, Z_{n}\right) \in S_{\succ}$, then for every positive integer $p$, the tuple $p \cdot Z$ also belongs to the set $S_{\succ}$, and

- if $Z=\left(Z_{1}, \ldots, Z_{n}\right) \in S_{\prec}$, then for every positive integer $p$, the tuple $p \cdot Z$ also belongs to the set $S_{\prec}$.

$12^{\circ}$. Let us prove that for every rational number $r=\frac{p}{q}$, where $p$ is an integer and $q$ is a positive integer, if a tuple $Z$ belongs to the set $S_{\sim}$, then the tuple $r \cdot Z$ also belongs to the set $S_{\sim}$.

Indeed, according to Part $10, Z \in S_{\sim}$ implies that $p \cdot Z \in S_{\sim}$.

According to Part 4 , for the tuple $r \cdot Z$, we have either $r \cdot Z \in S_{\sim}$, or $r \cdot Z \in S_{\succ}$, or $r \cdot Z \in S_{\prec}$.

- If $r \cdot Z \in S_{\succ}$, then, by Part 11 , we would get $p \cdot Z=q \cdot(r \cdot Z) \in S_{\succ}$, which contradicts our result that $p \cdot Z \in S_{\sim}$.

- Similarly, if $r \cdot Z \in S_{\prec}$, then, by Part 11 , we would get $p \cdot Z=q \cdot(r \cdot Z) \in S_{\prec}$, which contradicts our result that $p \cdot Z \in S_{\sim}$.

Thus, the only remaining option is $r \cdot Z \in S_{\sim}$. The statement is proven.

$13^{\circ}$. Let us now use continuity to prove that for every real number $x$, if a tuple $Z$ belongs to the set $S_{\sim}$, then the tuple $x \cdot Z$ also belongs to the set $S_{\sim}$.

Indeed, a real number $x$ can be represented as a limit of rational numbers: $r^{(k)} \rightarrow$ $x$. According to Part 12 , for every $k$, we have $r^{(k)} \cdot Z \in S_{\sim}$, i.e., the tuple $Z^{(k)} \stackrel{\text { def }}{=}$ $\left(\exp \left(r^{(k)} \cdot Z_{1}\right), \ldots, \exp \left(r^{(k)} \cdot Z_{n}\right)\right) \sim(1, \ldots, 1)$. In particular, this means that $Z^{(k)} \succeq$ $(1, \ldots, 1)$. In the limit, $Z^{(k)} \rightarrow\left(\exp \left(x \cdot Z_{1}\right), \ldots, \exp \left(x \cdot Z_{n}\right)\right) \succeq(1, \ldots, 1)$. By definition of the sets $S_{\sim}$ and $S_{\succ}$, this means that $x \cdot Z \in S_{\sim}$ or $x \cdot Z \in S_{\succ}$.

Similarly, for $-(x \cdot Z)=(-x) \cdot Z$, we conclude that $-x \cdot Z \in S_{\sim}$ or $(-x) \cdot Z \in S_{\succ}$. If we had $x \cdot Z \in S_{\succ}$, then by Part 9 we would get $(-x) \cdot Z \in S_{\prec}$, a contradiction. Thus, the case $x \cdot Z \in S_{\succ}$ is impossible, and we have $x \cdot Z \in S_{\sim}$. The statement is proven.

$14^{\circ}$. According to Parts 5 and 13, the set $S_{\sim}$ is closed under addition and under multiplication by an arbitrary real number. Thus, if tuples $Z, \ldots, Z^{\prime}$ belong to the set $S_{\sim}$, their arbitrary linear combination $x \cdot Z+\ldots+x^{\prime} \cdot Z^{\prime}$ also belongs to the set $S_{\sim}$. So, the set $S_{\sim}$ is a linear subspace of the $n$-dimensional space of all the tuples.

$15^{\circ}$. The subspace $S_{\sim}$ cannot coincide with the entire $n$-dimensional space, because then the pre-ordering relation would be trivial. Thus, the dimension of this subspace 
must be less than or equal to $n-1$. Let us show that the dimension of this subspace is $n-1$.

Indeed, let us assume that the dimension is smaller than $n-1$. Since the preordering is non-trivial, there exist tuples $y=\left(y_{1}, \ldots, y_{n}\right)$ and $y^{\prime}=\left(y_{1}^{\prime}, \ldots, y_{n}^{\prime}\right)$ for which $y \succ y^{\prime}$ and thus, $Z=\left(Z_{1}, \ldots, Z_{n}\right) \in S_{\succ}$, where $Z_{i}=\ln \left(\frac{y_{i}}{y_{i}^{\prime}}\right)$. From $Z \in S_{\succ}$, we conclude that $-Z \in S_{\prec}$.

Since the linear space $S_{\sim}$ is a less than $(n-1)$-dimensional subspace of an $n$ dimensional linear space, there is a path connecting $Z \in S_{\succ}$ and $-Z \in S_{\prec}$ which avoids $S_{\sim}$. In mathematical terms, this path is a continuous mapping $\gamma:[0,1] \rightarrow R^{n}$ for which $\gamma(0)=Z$ and $\gamma(1)=-Z$. Since this path avoids $S_{\sim}$, every point $\gamma(t)$ on this path belongs either to $S_{\succ}$ or to $S_{\prec}$.

Let $\bar{t}$ denote the supremum (least upper bound) of the set of all the values $t$ for which $\gamma(t) \in S_{\succ}$. By definition of the supremum, there exists a sequence $t^{(k)} \rightarrow \bar{t}$ for which $\gamma\left(t^{(k)}\right) \in S_{\succ}$. Similarly to Part 13, we can use continuity to prove that in the limit, $\gamma(\bar{t}) \in S_{\succ}$ or $\gamma(\bar{t}) \in S_{\sim}$. Since the path avoids the set $S_{\sim}$, we thus get $\gamma(\bar{t}) \in S_{\succ}$.

Similarly, since $\gamma(1) \notin S_{\succ}$, there exists a sequence $t^{(k)} \downarrow \bar{t}$ for which $\gamma\left(t^{(k)}\right) \in S_{\prec}$. We can therefore conclude that in the limit, $\gamma(\bar{t}) \in S_{\succ}$ or $\gamma(\bar{t}) \in S_{\sim}$ - a contradiction with our previous conclusion that $\gamma(\bar{t}) \in S_{\succ}$.

This contradiction shows that the linear space $S_{\sim}$ cannot have dimension $<n-1$ and thus, that this space have dimension $n-1$.

$16^{\circ}$. Every $(n-1)$-dimensional linear subspace of an $n$-dimensional superspace separates the superspace into two half-spaces. Let us show that one of these half-spaces is $S_{\succ}$ and the other is $S_{\prec}$.

Indeed, if one of the subspaces contains two tuples $Z$ and $Z^{\prime}$ for which $Z \in S_{\succ}$ and $Z^{\prime} \in S_{\prec}$, then the line segment $\gamma(t)=t \cdot Z+(1-t) \cdot Z^{\prime}$ containing these two points also belongs to the same subspace, i.e., avoids the set $S_{\sim}$. Thus, similarly to Part 15 , we would get a contradiction.

So, if one point from a half-space belongs to $S_{\succ}$, all other points from this subspace also belong to the set $S_{\succ}$. Similarly, if one point from a half-space belongs to $S_{\prec}$, all other points from this subspace also belong to the set $S_{\prec}$.

$17^{\circ}$. Every $(n-1)$-dimensional linear subspace of an $n$-dimensional space has the form $\alpha_{1} \cdot Z_{1}+\ldots+\alpha_{n} \cdot Z_{n}=0$ for some real values $\alpha_{i}$, and the corresponding halfspaces have the form $\alpha_{1} \cdot Z_{1}+\ldots+\alpha_{n} \cdot Z_{n}>0$ and $\alpha_{1} \cdot Z_{1}+\ldots+\alpha_{n} \cdot Z_{n}<0$.

The set $S_{\succ}$ coincides with one of these subspaces. If it coincides with the set of all tuples $Z$ for which $\alpha_{1} \cdot Z_{1}+\ldots+\alpha_{n} \cdot Z_{n}<0$, then we can rewrite it as

$$
\left(-\alpha_{1}\right) \cdot Z_{1}+\ldots+\left(-\alpha_{n}\right) \cdot Z_{n}>0
$$

i.e., as $\alpha_{1}^{\prime} \cdot Z_{1}+\ldots+\alpha_{n}^{\prime} \cdot Z_{n}>0$ for $\alpha_{i}^{\prime}=-\alpha_{i}$.

Thus, without losing generality, we can conclude that the set $S_{\succ}$ coincides with the set of all the tuples $Z$ for which $\alpha_{1} \cdot Z_{1}+\ldots+\alpha_{n} \cdot Z_{n}>0$. We have mentioned that

$$
y^{\prime}=\left(y_{1}^{\prime}, \ldots, y_{n}^{\prime}\right) \succ y=\left(y_{1}, \ldots, y_{n}\right) \Leftrightarrow\left(Z_{1}, \ldots, Z_{n}\right) \in S_{\succ},
$$


where $Z_{i}=\ln \left(\frac{y_{i}^{\prime}}{y_{i}}\right)$. Thus,

$$
y^{\prime} \succ y \Leftrightarrow \alpha_{1} \cdot Z_{1}+\ldots+\alpha_{n} \cdot Z_{n}=\alpha_{1} \cdot \ln \left(\frac{y_{1}^{\prime}}{y_{1}}\right)+\ldots+\alpha_{n} \cdot \ln \left(\frac{y_{n}^{\prime}}{y_{n}}\right)>0 .
$$

Since $\ln \left(\frac{y_{i}^{\prime}}{y_{i}}\right)=\ln \left(y_{i}^{\prime}\right)-\ln \left(y_{i}\right)$, the last inequality is equivalent to

$$
\alpha_{1} \cdot \ln \left(y_{1}^{\prime}\right)+\ldots+\alpha_{n} \cdot \ln \left(y_{n}^{\prime}\right)>\alpha_{1} \cdot \ln \left(y_{1}\right)+\ldots+\alpha_{n} \cdot \ln \left(y_{n}\right) .
$$

Let us take exp of both sides; then, due to the monotonicity of the exponential function, we get an equivalent inequality

$$
\exp \left(\alpha_{1} \cdot \ln \left(y_{1}^{\prime}\right)+\ldots+\alpha_{n} \cdot \ln \left(y_{n}^{\prime}\right)\right)>\exp \left(\alpha_{1} \cdot \ln \left(y_{1}\right)+\ldots+\alpha_{n} \cdot \ln \left(y_{n}\right)\right) .
$$

Here,

$$
\exp \left(\alpha_{1} \cdot \ln \left(y_{1}^{\prime}\right)+\ldots+\alpha_{n} \cdot \ln \left(y_{n}^{\prime}\right)\right)=\exp \left(\alpha_{1} \cdot \ln \left(y_{1}^{\prime}\right)\right) \cdot \ldots \cdot \exp \left(\alpha_{n} \cdot \ln \left(y_{n}^{\prime}\right)\right),
$$

where for every $i, e^{\alpha_{i} \cdot z_{i}}=\left(e^{z_{i}}\right)^{\alpha_{i}}$, with $z_{i} \stackrel{\text { def }}{=} \ln \left(y_{i}^{\prime}\right)$, implies that

$$
\exp \left(\alpha_{i} \cdot \ln \left(y_{i}^{\prime}\right)\right)=\left(\exp \left(\ln \left(y_{i}^{\prime}\right)\right)\right)^{\alpha_{i}}=\left(y_{i}^{\prime}\right)^{\alpha_{i}}
$$

so

$$
\exp \left(\alpha_{1} \cdot \ln \left(y_{1}^{\prime}\right)+\ldots+\alpha_{n} \cdot \ln \left(y_{n}^{\prime}\right)\right)=\left(y_{1}^{\prime}\right)^{\alpha_{1}} \cdot \ldots \cdot\left(y_{n}^{\prime}\right)^{\alpha_{n}}
$$

and similarly,

$$
\exp \left(\alpha_{1} \cdot \ln \left(y_{1}\right)+\ldots+\alpha_{n} \cdot \ln \left(y_{n}\right)\right)=y_{1}^{\alpha_{1}} \cdot \ldots \cdot y_{n}^{\alpha_{n}} .
$$

Thus, the condition $y^{\prime} \succ y$ is equivalent to

$$
\prod_{i=1}^{n} y_{i}^{\alpha_{i}}>\prod_{i=1}^{n}\left(y_{i}^{\prime}\right)^{\alpha_{i}}
$$

Similarly, we prove that

$$
\left(y_{1}, \ldots, y_{n}\right) \sim y^{\prime}=\left(y_{1}^{\prime}, \ldots, y_{n}^{\prime}\right) \Leftrightarrow \prod_{i=1}^{n} y_{i}^{\alpha_{i}}=\prod_{i=1}^{n}\left(y_{i}^{\prime}\right)^{\alpha_{i}} .
$$

The condition $\alpha_{i}>0$ follows from our assumption that the pre-ordering is monotonic.

$18^{\circ}$. Now, from permutation-invariance, we conclude that all the coefficients $\alpha_{i}$ are equal to each other: $\alpha_{i}=\alpha$ for all $i$. Thus, the condition $y^{\prime} \succ y$ is equivalent to 


$$
\prod_{i=1}^{n} y_{i}^{\alpha}=\left(\prod_{i=1}^{n} y_{i}\right)^{\alpha}>\prod_{i=1}^{n}\left(y_{i}^{\prime}\right)^{\alpha}=\left(\prod_{i=1}^{n} y_{i}^{\prime}\right)^{\alpha},
$$

i.e., to

$$
\prod_{i=1}^{n} y_{i}>\prod_{i=1}^{n} y_{i}^{\prime} .
$$

Similarly, we can prove that the condition $y^{\prime} \sim y$ is equivalent to

$$
\prod_{i=1}^{n} y_{i}^{\alpha}=\left(\prod_{i=1}^{n} y_{i}\right)^{\alpha}=\prod_{i=1}^{n}\left(y_{i}^{\prime}\right)^{\alpha}=\left(\prod_{i=1}^{n} y_{i}^{\prime}\right)^{\alpha},
$$

i.e., to

$$
\prod_{i=1}^{n} y_{i}=\prod_{i=1}^{n} y_{i}^{\prime}
$$

This completes the proof of our main result. 